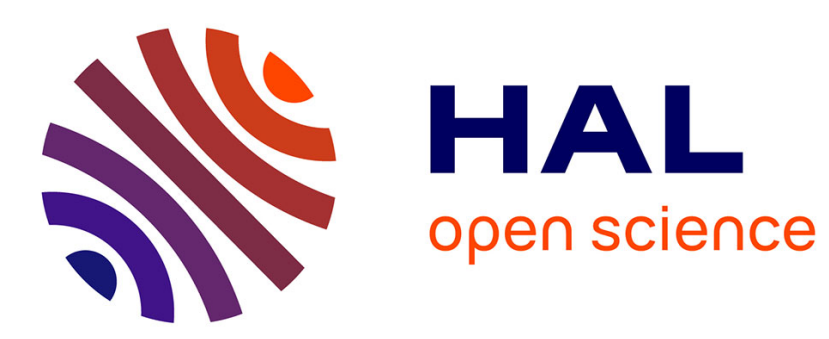

\title{
La quête du doctorat comme vecteur d'analyse de la professionnalisation du travail social
}

Stéphane Rullac

\section{To cite this version:}

Stéphane Rullac. La quête du doctorat comme vecteur d'analyse de la professionnalisation du travail social. Les Politiques Sociales, 2019, 100 ans de professionnalisation du travail social Enjeux situés et défis, 1-2. hal-02297797

\section{HAL Id: hal-02297797 \\ https://hal.science/hal-02297797}

Submitted on 26 Sep 2019

HAL is a multi-disciplinary open access archive for the deposit and dissemination of scientific research documents, whether they are published or not. The documents may come from teaching and research institutions in France or abroad, or from public or private research centers.
L'archive ouverte pluridisciplinaire HAL, est destinée au dépôt et à la diffusion de documents scientifiques de niveau recherche, publiés ou non, émanant des établissements d'enseignement et de recherche français ou étrangers, des laboratoires publics ou privés. 


\section{La quête du doctorat comme vecteur d'analyse de la}

professionnalisation du travail

\section{social}

\section{Stéphane Rullac}

Professeur en innovation sociale à la Haute École de Travail Social et de la Santé de Lausanne (EESP), Haute École Spécialisée de Suisse Occidentale (HES-SO).

\section{Le fait doctoral est un catalyseur de professionnalisation}

\subsection{Un marqueur d'autonomie}

Le doctorat $n^{\prime}$ est pas souvent étudié en tant que fait social. II est la plupart du temps perçu comme un outil neutre pour "faire de la science ", et comme un simple vecteur à analyser au regard des apports de savoirs qu'il contribue à produire pour sa discipline de référence. Pourtant, avant d'être un outil pour faire avancer la science, le doctorat est aussi un marqueur de professionnalisation. Le docteur est un expert scientifique reconnu par l'obtention du plus haut grade universitaire dans le cadre d'une discipline donnée. II y a ceux qui sont docteurs et ceux qui ne le sont pas, mais il y a aussi les professions qui disposent d'un tel grade universitaire et celles qui n'en disposent pas.

Je postule l'existence d'une symétrie de légitimation entre les individus et les professions. Si le fait d'être docteur octroie une légitimité individuelle à être reconnu en tant que scientifique dans une discipline, à l'échelle des professions le fait de disposer de ce grade octroie une légitimité en tant que science (1). Les professions qui incorporent une scientifisation de leurs acteurs sont intégrées au cercle très fermé des collectifs autorisés à contribuer aux débats sociétaux. Dans un deuxième cercle, les autres professions, qui ne disposent pas de cette habilitation, se trouvent limitées dans leur expertise à produire au mieux de la technologie - dans le cadre de savoirs orientés vers la pratique - selon 


\section{Les politiques sociales}

\section{$1 \& 2 / 2019$}

les théories développées par les savoirs scientifiques. Enfin, dans un troisième cercle, la multitude de pratiques n'est pas considérée comme constituant une profession, et chacune de ces dernières applique les techniques que ses métiers ont développées.

Enfin, dans un troisième cercle, la multitude de pratiques n'est pas considérée comme constituant une profession, et chacune de ces dernières applique les techniques que ses métiers ont développées.

$C^{\prime}$ est dans cette pyramide des professionnalisations (professions scientifiques, professions technologiques et métiers techniques (2) que se situe le fait social dont le doctorat est la pierre angulaire; cela dans le cadre d'une construction sociale des activités professionnelles dont la légitimité est construite sur une dichotomie entre théorie et pratique. $C^{\prime}$ est ainsi que les professions scientifiques éclairent de leurs théories les professions technologiques, qui déterminent à leur tour les techniques des métiers. Cette hiérarchie se déploie de situation d'autonomie, en haut, vers celle d'hétéronomie, en bas.

$C^{\prime}$ est à ce titre que le fait de posséder un doctorat serait un critère objectif d'appartenance à l'élite en matière de professionnalisation.

\subsection{Une légitimité recherchée mais à double tranchant}

L'existence d'un doctorat est le marqueur d'une autonomie en matière de professionnalisation, à penser pour soi et pour les autres. La lutte pour obtenir la création d'un doctorat au sein d'un champ professionnel constitue donc à la fois une force symbolique à "dire " le sociétal, et une force pragmatique à déterminer les technologies et les techniques des autres types de champs professionnels. Le fait de disposer d'un doctorat, et donc de la maîtrise d'une discipline scientifique, est logiquement très recherché pour accéder à ce type de professionnalisation particulièrement valorisante.

$C^{\prime}$ est dans cette logique que I'on comprend mieux le combat des ingénieurs et des infirmiers à développer un doctorat, afin de sortir de leur expertise praticienne comme unique vecteur de légitimité professionnelle. En obtenant le statut de science, l'enjeu est de pouvoir penser de manière endogène ses pratiques et de réduire une situation $\mathrm{d}$ 'hétéronomie (comme par exemple la médecine), mais aussi de développer une forme de domination par rapport aux autres professions non scientifiques et à tous les métiers du même champ d'activité. C'est ce que résume de manière éloquente le titre $d$ 'un article récent qui plaide pour le développement des sciences infirmières : Une profession forte 
La quête du doctorat comme vecteur d'analyse de la professionnalisation du

travail social

par une discipline forte et une discipline forte par une profession forte (Lecordier et al., 2018).

Pourtant, cette construction sociale est problématique à plusieurs niveaux. D'un point de vue épistémologique, la dichotomie entre théorie et pratique est douteuse, mais concrètement, une identité professionnelle basée exclusivement sur une légitimité théorique peut constituer un frein pour sa reconnaissance effective en tant que profession. C'est ce qu'explique François Aballéa au sujet de la sociologie (Aballéa, 2007, p. 109-110) : "La professionnalisation de la sociologie est une question ancienne dans le champ de la discipline. En réalité, les sociologues ont, depuis l'origine, été pris dans une tension et confrontés à une tentation. La tension, c'est celle qui oppose le savoir à l'action ou la connaissance à l'intervention. [...] Le statut de la sociologie se dessine ainsi comme un savoir utile à l'action, non comme un savoir en action. La professionnalisation de la sociologie ne s'est pas faite ". C'est ainsi que la sociologie bénéfice de la plus haute reconnaissance en tant que profession, grâce au support de la discipline et de la science qu'elle produit, mais que les sociologues ne bénéficient pas d'une importante reconnaissance professionnelle en dehors des universités. Le fait de posséder une reconnaissance scientifique grâce au doctorat est donc un atout considérable pour faire valoir une pensée, mais ne dispense pas pour autant de faire valoir une technologie et des techniques. La tentation des sociologues est de se situer du côté des "sachants " et non des acteurs, de peur d'être assimilés à des activités techniques situées en bas de la pyramide des légitimités professionnelles.

À I'heure où le travail social cherche à dépasser une reconnaissance originelle technicienne pour proposer une expertise technologique (3) (Vulbeau, 2011), et aspire au développement d'un savoir scientifique (Rullac, 2012), l'exemple de la sociologie n'est pas à prendre à la légère. Si l'obtention d'un doctorat en travail social constituerait un mode de professionnalisation complet, le renoncement à une identité praticienne préalable constitue quant à lui un faux-semblant en matière de légitimation. C'est ce que la médecine à très bien compris depuis ses origines. 


\section{Le travail social est le parangon d'une professionnalisation en manque de légitimé}

\subsection{Une recherche structurelle de reconnaissance d'expertise}

Le développement du travail social semble historiquement se construire sur une quête de reconnaissance en tant que profession à part entière, construite sur une science au service de la professionnalisation. Dans certains pays (notamment la France, la Belgique et la Suisse) cette quête est encore inachevée - voire quasi impossible en France - en ce qui concerne la création d'un doctorat en travail social, qui représente encore un point de butée. Ma réflexion en la matière s'est développée à partir de trois projets distincts :

1) Une HDR en sociologie soutenue et publiée en 2014, portant sur les enjeux de la quête d'une discipline du travail social en France (Rullac, 2014).

2) La co-organisation d'un Forum international au Conservatoire National des Arts et Métiers (CNAM) de Paris, les 1, 2 et 3 février 2017, portant sur "Les "défis" de la création du doctorat de travail social ".

3) La codirection d'un ouvrage collectif intitulé La fabrique $d u$ doctorat en travail social, publié en 2018 (Rullac, Tabin, Frauenfelder, 2018).

Ces initiatives $\mathrm{m}^{\prime}$ ont permis de développer une analyse concernant la difficulté propre à ce champ professionnel - à l'échelle mondiale - à être reconnu comme suffisamment homogène dans ses références pour obtenir la reconnaissance suprême d'une profession scientifique. Sorti péniblement de la nasse des métiers techniques, le travail social a tendance à stagner dans sa reconnaissance en tant que profession technologique, qui se voit dès lors dicter la théorie de ses pratiques par les autres sciences humaines et sociales.

Partout dans le monde, la création d'un doctorat de travail social a été un combat généralement récent, et demeure un défi pour faire valoir la légitimité de la théorie produite. Pour le dire simplement, un docteur en travail social à très souvent tendance à être considéré comme un sociologue raté, et souffre de sa place minoritaire dans I'Université (4). Aux États-Unis par exemple, si le doctorat de travail social existe, ce diplôme est parfois totalement absent dans certains États. Et dans la plupart des pays, la question de l'utilité d'un tel diplôme pour les 


\section{La quête du doctorat comme vecteur d'analyse de la professionnalisation du}

travail social

interventions de ce champ professionnel ne fait pas l'unanimité, en dehors de la trajectoire vers une carrière universitaire.

Cette difficulté à asseoir une théorie de ses pratiques condamne le travail social à ne pas être reconnu comme une profession de premier plan, autorisée à "dire " la réalité sociale du haut de son expertise scientifique. Au début du $X X I^{e}$ siècle, ce constat est l'indication d'un échec de ce champ professionnel à dépasser les freins sociétaux et endogènes à la profession qui limitent cette reconnaissance. Pour mesurer cet état de fait, il suffit de relire les élans formulés pour définir le travail social lors de la Première conférence internationale du service social de 1928, à Paris. C'est à cette occasion que l'un des représentants français, le $\mathrm{Dr}$ Pierre Joannon, formulait des ambitions dont il est intéressant de mesurer la réalité près d'un siècle plus tard : "Le service social est l'utilisation judicieuse et dévouée de moyens préventifs et curatifs de lutte contre un grand nombre de maux sociaux, ces moyens de lutte constituant I'armement social. [...] Tout d'abord, les travailleurs sociaux s'y livrent en dehors du service social proprement dit, c'est-àdire de l'exercice même de leur profession - le mot profession devant naturellement être pris ici dans son acception la plus noble. [...] À son sujet, on parle, non sans raison, de religion terrestre, de science, d'art. " (Vol. 1, p. 172-173.)

Pour bien mesurer I'hiatus entre 1928 et 2018, la situation française est édifiante.

\subsection{Le cas français est l'idéal-type de l'échec permanent}

Malgré de nombreuses mobilisations, chaque génération a échoué à faire reconnaître le travail social comme une profession de premier plan, qui serait soutenue par une discipline scientifique, comme le droit, la psychologie ou les sciences de l'éducation en France. Citons tout d'abord les promoteurs de la recherche en travail social (Bouquet, Duchamp, Drouard, 1989) qui ne sont pas parvenus à faire valoir leur épistémologie d'une science du travail social. Malgré tout, ces pionniers ont su créer un mouvement associatif qui subsiste encore, en créant I'Association française de recherche en travail social (I'AFFUTS), à I'origine notamment de la création de la chaire du travail social au CNAM de Paris - dont la première professeure titulaire a été Brigitte Bouquet en 2001 (Bouquet, 2006). Elle est devenue la Chaire du travail social et de I'intervention sociale avant la prise de fonction de Marcel Jaeger en 2009 comme deuxième professeur titulaire. Ce dernier a organisé une Conférence de consensus les 14 et 15 novembre 2012, intitulée " La 
recherche en/dans/sur le travail social " (Jaeger, 2014). Dans la foulée, le CNAM de Paris a créé en 2013 deux spécialités doctorales dans les disciplines de sociologie et des sciences de l'éducation.

Dans cette même dynamique, le gouvernement a initié un Plan $d^{\prime}$ action en faveur du travail social et de développement social, dans le cadre des États Généraux du Travail Social, publié à la suite du Conseil des Ministres du 21 octobre 2015 ; ce Plan affirme qu' " à moyen terme, l'objectif pourrait être la constitution d'une discipline universitaire en travail social " (p. 26). En 2018, une réforme des Diplômes d'État (DE) du travail social a été entreprise pour octroyer aux diplômes concernés le grade de Licence, conformément à I'harmonisation européenne de Bologne. La perspective envisagée un temps de créer - comme presque partout dans le monde - un diplôme unifié de "travailleur social ", avec des options, a échoué sous la pression des organisations professionnelles qui ne voulaient pas que soient dépassées les spécificités des cinq métiers concernés (5). À I'issue de la réforme, ces DE demeurent distincts - même si un tiers de la formation est commune - et sont accompagnés par les neuf autres titres professionnels français de ce champ professionnel. En 2018, en France, le travail social n'est toujours pas une discipline, le doctorat $n^{\prime}$ existe pas davantage (aucune autre université n'a suivi a minima l'initiative du CNAM de créer des spécialités " travail social ») - et la Chaire du CNAM est toujours la seule en France à exister sous cette appellation générique.

Au regard de ces éléments, le travail social en 2018 en France n'est pas une religion, ni une science, mais peut-être un art qui le condamne à ne pas être une profession mais une galaxie de métiers, contrairement aux vœux du Dr Joannon en 1928.

\section{Quelques enjeux de professionnalisation du travail social du développement d'un doctorat}

\subsection{Caractérisation des freins dans un processus de disciplinarisation secondaire}

La difficulté structurelle du travail social, à l'échelle mondiale, de créer puis de légitimer une discipline scientifique - et par la même occasion un doctorat qui en est le principal outil de développement - est liée au phénomène de "disciplinarisation secondaire ". Ce phénomène est une caractéristique des professions qui ont développé une pratique professionnelle avant de faire reconnaître leurs théories de la pratique à 


\section{La quête du doctorat comme vecteur d'analyse de la professionnalisation du}

travail social

travers une discipline (Hofstetter, Schneuwly, 2001). Dans le cadre d'une disciplinarisation secondaire, comme le travail social, trois écueils se présentent :

- articuler la finalité de la profession avec celle de la discipline qui s'y rattache, dans le cadre d'une tentation à distinguer les deux ;

- saisir les pratiques professionnelles de manière scientifique, dans le cadre d'une tentation de théoriser l'impossibilité de faire émerger un savoir scientifique à partir d'un savoir professionnel ;

- mener une approche nécessairement interdisciplinaire dans le cadre d'une tentation à critiquer l'absence de cohérence du corpus mobilisé.

Toutes les disciplinarisations secondaires (comme par exemple les sciences de l'éducation bâties sur l'enseignement scolaire en France) impliquent une tentation - généralisée parmi les disciplines antérieurement créées - de mettre en question la légitimité du processus de scientifisation qui se déroule sous leurs yeux. L'inclinaison est alors forte de mettre en œuvre un processus " d'englobement du contraire ", défini par les juristes comme une stratégie de défense relevant de I'ethnocentrisme et qui consiste à opposer à son adversaire l'illusion qu'une seule réalité existe ; cela conformément au « mythe de la Raison universelle, que partagent tous les êtres humains et qui doit donc forcément les mener à aboutir aux mêmes conclusions, à une même vision rationnelle de la réalité et de son organisation " (Eberhard, 2012, p. 175). Ainsi, chaque discipline préexistante oppose aux prétendantes ses propres références (épistémologiques, conceptuelles et méthodologiques), dans l'optique de discréditer l'innovation scientifique apportée par le nouveau paradigme émergent. La création d'un doctorat en travail social se trouve donc aux prises avec un processus généralisé de procès en illégitimité de la part des sciences préétablies, bien éloigné de la rationalité scientifique de vérification des hypothèses proposées par un éventuel nouveau champ disciplinaire.

Pour résister à cette charge menée par les savoirs établis, il faut être particulièrement forts, soudés et cohérents dans les arguments avancés, afin de remporter finalement ce qui pourrait se révéler être une révolution scientifique ; atouts que le travail social ne parvient manifestement pas à rassembler parfaitement, à l'échelle mondiale. La compréhension de cette caractéristique apparemment structurelle serait un excellent sujet de recherche, pour comprendre comment ce trait parvient à transcender 
I'hétérogénéité du travail social à l'échelle nationale ; il est dommage que ce point commun représente une faiblesse.

\subsection{Lutte corporatiste entre sciences normales et sciences extraordinaires}

La création d'une discipline, et donc d'un doctorat, n'est pas qu'une affaire scientifique. C'est aussi un enjeu de professionnalisation du champ académique ; or celui-ci a tendance à souhaiter le statu quo, dans une logique corporatiste, pour ne pas ouvrir cet empire terrestre du savoir scientifique à d'autres prétendants et leur octroyer les privilèges qui vont avec. L'une des stratégies corporatistes des disciplines préexistantes, qui s'intègre dans la logique de l'englobement du contraire, tient justement à faire croire que leur propre création repose exclusivement sur un consensus scientifique, alors que la dimension sociale est aussi - voire davantage - importante pour déclencher le nécessaire désir social en dehors des murs de l'Université (Karady, 1979). Thomas Kuhn a permis de penser ces processus, dans une approche fonctionnaliste très utile pour les militants (dont je fais partie) de la disciplinarisation (6) du travail social. Pour ce dernier, une discipline bien établie universitairement est une science normale, qui fait état d'une maturité dans le traitement scientifique de son objet, selon les références stabilisées qui reposent avant tout sur la reproductibilité dans une logique corporatiste. " La science normale n'a jamais pour but de mettre en lumière des phénomènes d'un genre nouveau; ceux qui ne cadrent pas avec la boîte passent même souvent inaperçus. Les scientifiques n'ont pas non plus pour but, normalement, d'inventer de nouvelles théories, et ils sont souvent intolérants envers celles qu'inventent les autres. Au contraire, la recherche de la science normale est dirigée vers I'articulation des phénomènes et des théories que le paradigme fournit déjà. " (Kuhn, 1983, p. 47.) Une science normale est la reconnaissance pleine et entière d'un paradigme scientifique qui permet de former, définir et institutionnaliser une communauté scientifique sur la base de "I'ensemble de croyances, de valeurs reconnues et de techniques qui sont communes aux membres d'un groupe donné " (Kuhn, 1983, p. 238). En dehors de ces paradigmes scientifiques stabilisés, il en existe d'autres qui ne sont pas parvenus à un tel degré de légitimité et forment ce que Kuhn appelle les sciences extraordinaires. Toujours d'après la théorie des révolutions scientifiques de Kuhn, l'état de maturité d'un paradigme scientifique repose sur l'existence d'une matrice disciplinaire stabilisée et légitime, composée de plusieurs éléments : 


\section{La quête du doctorat comme vecteur d'analyse de la professionnalisation du}

travail social

- généralisations symboliques: ce sont les affirmations logiques acquises comme évidentes par les membres du groupe, qui s'apparentent de près ou de loin à des lois scientifiques, mais aussi à des définitions de concepts à la base des lois ;

- la partie métaphysique : ce sont les croyances qui fondent des modèles de pensée. Ce sont les bases qui permettent de fonder les accords, les désaccords et les problèmes qui résistent à la résolution ;

- les valeurs : ce sont des affirmations larges qui permettent de faire naître le sentiment d'appartenance à un groupe ;

- les exemples : ce sont des cas résolus qui permettent aux étudiants de se former et d'acquérir les trois éléments précités.

Au regard de ces éléments, le travail social se situe historiquement du côté des sciences extraordinaires, du fait de sa difficulté à montrer la solidité de sa matrice disciplinaire. D'un point de vue anthropologique, celle-ci compose les caractéristiques d'une culture commune, selon des références ou traits culturels qui permettent à des chercheurs de faire communauté en se reconnaissant l'appartenance à un même groupe, selon des bases acquises et reproduites. L'enjeu actuel pour la professionnalisation du travail social se situe donc dans sa capacité à créer enfin en son sein une communauté scientifique qui d'une part soit réunie autour de son objet professionnel et de sa capacité à construire un corpus de références (épistémologiques, conceptuelles et méthodologiques); d'autre part puisse s'imposer comme légitime en interne, puis en externe par rapport aux autres disciplines - mais aussi plus largement aux yeux de la société toute entière.

La création d'une matrice disciplinaire est l'un des principaux défis en matière de professionnalisation du travail social en ce début de $\mathrm{XXl}^{\mathrm{e}}$ siècle, défi qui s'avère particulièrement ardu dans le cadre d'une disciplinarisation secondaire.

\subsection{Spécificités épistémologiques d'un doctorat en travail social}

À quoi un doctorat en travail social peut-il servir pour ce champ professionnel ? Cette question, qui concerne finalement l'utilité professionnelle de cet outil académique, est capitale pour sa création mais surtout pour sa pérennisation.

En premier lieu, un doctorat en travail social doit être un outil qui permette aux professionnels : de sortir de leur empirisme radical, issu de I'histoire de leur champ d'activité à tradition non scientifique; et de 


\section{Les politiques sociales}

\section{$1 \& 2 / 2019$}

construire les outils d'objectivation collective qui leur permettent de dépasser leur identité professionnelle prise dans une assignation à la praticité, en tant qu'ingénieur et technicien de la relation (Rullac, 2014). En ce sens, l'outil doctoral est un vecteur pour créer des références discutées de manière collective à l'échelle du champ professionnel, pour que chaque acteur et institution puisse bénéficier des effets cumulatifs du débat scientifique, afin de relever les défis de la complexité de ses missions.

Ces références, même si elles se développent dans un champ disciplinaire, ne peuvent qu'être de nature pluridisciplinaire pour intégrer tous les types de savoirs indispensables à la saisie de la complexité du travail social; voire transdisciplinaire pour intégrer les savoirs des personnes bénéficiaires issus de l'expérience d'usage (Rullac, 2018). Et surtout, un doctorat de travail social doit s'affirmer comme un outil au service de l'innovation en travail social, afin de soutenir l'adaptation de ce champ professionnel aux évolutions sociétales auxquelles il se trouve confronté au quotidien, selon la définition suivante du Centre de Recherche sur les Innovations Sociales (Crises) : « [...] une intervention initiée par des acteurs sociaux, pour répondre à une aspiration, subvenir à un besoin, apporter une solution ou profiter d'une opportunité d'action afin de modifier des relations sociales, de transformer un cadre d'action ou de proposer de nouvelles orientations culturelles. "

Dans cette optique, l'épistémologie mise en œuvre n'a pas à prôner une rupture systématique avec le sens de l'action des professionnels ou des bénéficiaires, mais au contraire à permettre une réappropriation du sens de l'engagement dans l'expérience au sein du travail social, afin que chacun puisse étayer ses choix objectivement et en pleine conscience déontologique. En cela, le doctorat en travail social est un outil au service de l'autonomisation des acteurs et institutions du travail social, afin qu'ils soient en mesure de négocier le sens de leurs pratiques, entre le poids de leur subjectivité et celui des normes sociales. L'enjeu est que le travail social échappe aux logiques héritées de son passé, qui sont surdéterminées à la fois par le sens militant du travailleur social - mobilisé par ses valeurs individuelles - et par les prescriptions normatives d'une action financée le plus souvent par les pouvoirs publics ou les fondations privées.

En ce sens, le doctorat en travail social est un vecteur de développement de l'autonomie de ce champ professionnel, sur une ligne de crête qui se situe entre le risque de "l'adéquationnisme "- qui vise 
la soumission du contenu des savoirs aux prescriptions sociales (Pinto, 2008) - et la capacité à l'adaptation face aux problématiques sociales qui mutent en permanence. La recherche de cette frontière est fragile, mais constitue certainement le fil conducteur épistémologique commun à toutes les recherches scientifiques dans le champ du travail social, que l'outil doctoral doit soutenir. Enfin, les processus d'élaboration du doctorat en travail social ne peuvent pas faire l'impasse sur l'articulation des trois savoirs nécessaires à la résolution de la question du travail social : le savoir disciplinaire, le savoir professionnel et le savoir $d^{\prime}$ expertise d'usage des bénéficiaires. Cette articulation implique des méthodes collaboratives et plaide pour le développement d'identités hybrides au sein des processus de professionnalisation des acteurs du travail social - des travailleurs sociaux aux usagers, en passant par les partenaires - qui ont alors comme point commun $d$ 'appartenir simultanément au même terrain.

Finalement, le développement d'un paradigme scientifique du travail social dépasse largement les acteurs professionnels du travail social, et intègre également tous ceux qui en font usage. C'est sûrement cette dimension usagère qui constitue le principal défi en termes de professionnalisation dans le cadre du développement d'un doctorat en travail social.

\section{Bibliographie}

Aballéa, F. (2007). Les surintendantes d'usines: un échec de la professionnalisation de la sociologie. In Vie sociale, 2(4), 109-126.

Bouquet, B. (2006). La Chaire du travail social au CNAM. In Informations sociales, 7(135), 78-79.

Bouquet, B., Duchamp, M., \& Drouard, H. (1989). La Recherche en travail social. Paris : Centurion.

Eberhard C. (2012). Droits de l'Homme et Dialogue Interculturel. Paris : Connaissances et savoirs.

Hofstetter, R., \& Schneuwly, B. (dirs). (2001). Le pari des sciences de l'éducation. Louvain-la-Neuve, Belgique : De Boeck Université.

Jaeger, M. (dir.). (2014). Recherche et travail social. Conférence de consensus, Paris : Dunod. 


\section{Les politiques sociales}

\section{$1 \& 2 / 2019$}

Karady, V. (1979). Stratégies de réussite et modes de faire-valoir de la sociologie chez les durkheimiens. In Revue française de sociologie, 20(1).

Kuhn, T. (1983). La structure des révolutions scientifiques. Paris : Flammarion. Lecordier, D. et al. (2018). Une profession forte par une discipline forte et une discipline forte par une profession forte. In Recherche en soins infirmiers, 2(133), 5-6.

Lévesque, B. (2008). Le potentiel d'innovation et de transformation de l'économie sociale : quelques éléments de problématique. In Interações (Campo Grande), 9(2), 191-216.

Pinto, V. (2008). Démocratisation et professionnalisation de l'enseignement supérieur. In Mouvements, 3(55-56), 12-23. Première conférence internationale du service social: Paris, 8-13 juillet 1928. Volume 1. Paris : Gallica/Cédias Musée social.

Rullac, S. (2012). La science du travail social : Hypothèses et perspectives. Issyles-Moulineaux : ESF.

Rullac, S. (2014). Dépasser une assignation à la praticité. In M. Jaeger (dir.), Le travail social et la recherche. Conférence de consensus. Paris: Dunod, 117-133.

Rullac, S. (dir.). (2014). La scientifisation du travail social. Recherche en travail social et discipline universitaire. Rennes: Les Presses de l'EHESP, Politiques et interventions sociales.

Rullac, S. (2018). Recherche Action Collaborative en travail social : les enjeux épistémologiques et méthodologiques d'un bricolage scientifique. In Pensée plurielle, 2(48), 37-50.

Rullac, S., Tabin, J.-P., \& Frauenfelder, A. (2018). La fabrique du doctorat en travail social. Rennes : Les Presses de l'EHESP.

Vulbeau, A. (2011). Contrepoint - L'émergence de l'ingénierie sociale. In Informations sociales, 167(5), 51-51.

\section{Notes}

(1) Dans cet article, la discipline et la science seront utilisées comme les deux faces d'une même réalité : la discipline institutionnalise universitairement un savoir scientifique. 


\section{La quête du doctorat comme vecteur d'analyse de la professionnalisation du}

travail social

(2) Cette hiérarchie des professions se retrouve notamment dans la nomenclature des Professions et Catégories Socioprofessionnelles (PCS) utilisée par l'Institut National de la Statistique et des Études Économiques (INSEE) en France. C'est ainsi que trois catégories distinguent les salariés, selon un principe de catégorisation des activités allant des plus techniques au plus intellectuelles : le groupe 3, qui comprend les cadres, les ingénieurs, les professeurs de lycée général, et au-delà les catégories $\mathrm{A}$ de la fonction publique, les directeurs généraux et leurs adjoints directs ; le groupe 4, qui comprend les professions intermédiaires, les techniciens et techniciens administratifs, les agents de maîtrise, les contremaitres, les professeurs de lycée professionnel, et en dessous les infirmières, les assistantes sociales et les catégories B de la fonction publique; le groupe 5, qui comprend les employés de bureau, de commerce et des services, les aides-soignants et les catégories $\mathrm{C}$ de la fonction publique. Au même niveau on trouve le groupe 6, composé des ouvriers hautement qualifiés, qualifiés, non qualifiés et des manœuvres.

(3) Nous faisons ici référence au développement de la figure de l'ingénieur social dans ce champ professionnel. Comme par exemple en France, avec la création en 1978 du Diplôme Supérieur du Travail Social, devenu en 2005 le Diplôme d'État d'Ingénierie Sociale.

(4) $C f$. le texte édifiant de la situation en Italie, dans le chapitre écrit par Annamaria Campanini dans La Fabrique du doctorat, qui évoque la difficulté pour des docteurs en travail social à se faire embaucher comme professeurs dans leur propre discipline, au profit d'autres profils disciplinaires.

(5) Diplôme d'État d'Assistant de Service Social (DEASS), Diplôme d'État d'Éducateur Spécialisé (DEES), Diplôme d'État d'Éducateur de Jeunes Enfants (DEEJE), Diplôme d'État d'Éducateur Technique Spécialisé (DEETS), et Diplôme d'État de Conseiller en Économie Sociale Familiale (DECESF).

(6) Précisons que la disciplinarisation est le processus de création et de développement d'une discipline en tant que système. Évoquer une disciplinarisation du travail peut consister à envisager une création 


\section{Les politiques sociales}

$1 \& 2 / 2019$

disciplinaire mais aussi un champ disciplinaire thématique dans les disciplines préexistantes. 